
internationalen Fachliteratur referiert und kommentiert.

\section{Fraglicher Nutzen der Akupunktur bei allergischer Rhinitis}

\section{Deutsche Wissenschaftler haben untersucht, ob sich saisonale allergische Rhinitis mit Akupunkturnadeln behandeln lässt. Die Ergebnisse fielen zwar positiv aus. Doch die Effekte waren so klein, dass ihre klinische Relevanz fraglich ist.}

$\mathrm{N}$ eben Medizinern der Berliner Charité, darunter der Studienleiter Benno Brinkhaus, arbeiteten Ärzte von fünf weiteren Kliniken und 32 ambulanten Einrichtungen an der randomisierten und kontrollierten Studie mit. 422 Pollenallergiker nahmen teil. 212 von ihnen wurden ab Beginn des Birkenpollenflugs über einen Zeitraum von acht Wochen zwölfmal akupunk-
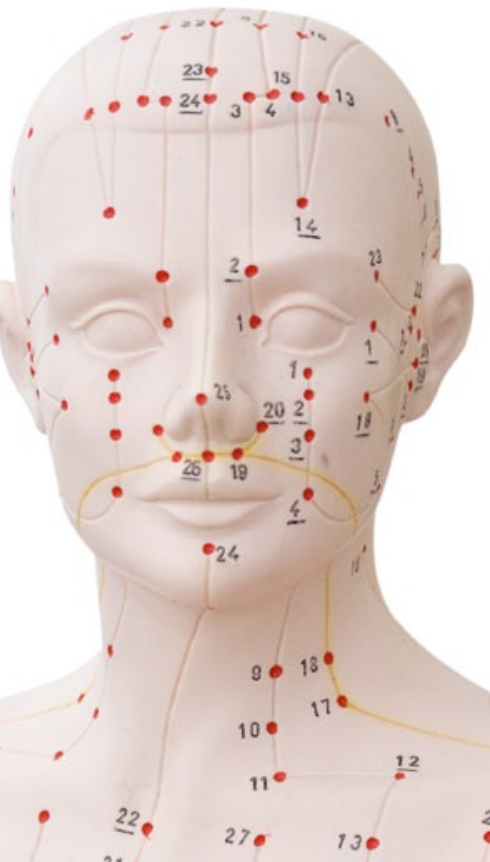

27.

$26 \cdot$

140
2

.5

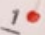

tiert. Zusätzlich zur Akupunkturbehandlung durften sie auf Cetirizin und gegebenenfalls orale Kortikoide als Bedarfsmedikation zurückgreifen. 102 Probanden erhielten eine Scheinakupunktur und die genannten Bedarfsmedikamente, 108 Teilnehmer wurden ausschließlich medikamentös behandelt. Am Ende der achtwöchigen Akupunkturphase, nach weiteren acht Wochen und nach einer achtwöchigen Vergleichsphase im Jahr darauf wurde der Therapieerfolg verglichen.

Als Messinstrument dienten primär das „Rhinitis Quality of Life Questionnaire" (RQLQ), das mit 28 Fragen aus sieben Bereichen etwaige Beeinträchtigungen abfragt. Der gebildete Mittelwert liegt zwischen 0 (keine Beeinträchtigung) und 6 (schwer beeinträchtigt). Gemessen wurde auch die durchschnittlich pro Woche nötige „Rettungsmedikation" (RM); die Punktwerte reichten hier von 0 (keine Medikation) bis 21 (täglich Kortikoide). Als Wirknachweis definierten die Forscher eine Verminderung des RQLQ-Wertes um 0,5 und eine Reduktion des RM-Wertes um 1,5 Punkte.

Zu Beginn der Studie hatten die RQLQ-Werte in der Akupunkturgruppe bei durchschnittlich 2,7, in der Scheinakupunkturgruppe bei 2,3 und in der Gruppe mit nur medikamentöser
Therapie bei 2,5 gelegen. Für RM schlugen im Schnitt 2,3, 2,6 und 3,2 Punkte zu Buche. Nach acht Wochen, also am Ende der Akupunkturphase, war der RQLQ-Score gesunken, und zwar um 1,0 (Akupunktur), 0,5 (Scheinakupunktur) bzw. 0,3 Punkte (Medikamente). Das ergab zwar eine signifikante Reduktion zugunsten der Akupunktur. Die Konfidenzintervalle waren allerdings so breit, dass der definierte Schwellenwert nicht sicher erreicht wurde. Für RM ergaben sich Minderungen um 1,5, 0,4 und 0,05 Punkte mit gleicher Einschränkung bezüglich der Intervallgrenzen.

Weitere acht Wochen später waren alle Unterschiede verschwunden. Nach der Kontrollphase im Jahr darauf zeigten sich signifikante, gleichwohl unterschwellige Vorteile der Akupunktur gegenüber der Scheinakupunktur. Die Differenzen zur alleinigen Medikation waren statistisch unbedeutend.

Fazit: Zumindest solange die Nadeln gesetzt werden, lässt sich rechnerisch an den Studienergebnissen also ein signifikanter Nutzen der Akupunktur bei saisonaler allergischer Rhinitis ablesen. „Die klinische Bedeutung dieser Resultate bleibt aber unklar", räumen die Forscher ein. Künftige Untersuchungen müssten klären, welche möglichen Mechanismen etwaigen Effekten zugrunde lägen - ein Satz, der Artikel über Akupunkturstudien so signifikant häufig wie bedeutungsunklar beschließt.

Dr. Robert Bublak

Brinkhaus B. Acupuncture in Patients With Seasonal Allergic Rhinitis. Ann Intern Med. 2013; 158: 225-34 